\title{
ANÁLISIS BIBLIOMÉTRICO DE LAS TESIS DE PREGRADO DE LA FACULTAD DE MEDICINA VETERINARIA DE LA UNIVERSIDAD NACIONAL MAYOR DE SAN MARCOS EN EL PERIODO 2001-2006
}

\author{
B ibliometric Analysis of Undergraduate Thesis in the F aCulty of Veterinary \\ Medicine, San Marcos National University, in the Period of 2001-2006
}

Felipe San Martín H. ${ }^{1.2}$ y Josmel Pacheco M. ${ }^{1}$

\section{Resumen}

\begin{abstract}
Las tesis de pregrado tienen gran importancia en la actividad de investigación de la Facultad de Medicina Veterinaria de la Universidad Nacional Mayor de San Marcos (FMV-UNMSM) por lo que se realizó el presente estudio, a fin de evaluar la actividad de investigación mediante un análisis bibliométrico de aquellas tesis que hayan sido sustentadas en el periodo 2001-2006. Se tomó en consideración las variables unidad académica, disciplina, especie animal, directores y jurado de tesis. Los datos se organizaron y normalizaron en una base de datos que registra las comunicaciones científicas de la FMV-UNMSM (REGULVS) y se usó Excel para la evaluación y confección de matrices de coincidencia utilizando la macro Microsoft Excel Toolinf $\odot$. Se registraron 247 tesis con una media anual de 41 . El mayor número de tesis involucró al canino (15.5\%) y bovino (15.5\%), seguido por aves (13.4\%), porcinos (12.6\%) y alpacas $(11.3 \%)$. El área de Salud Animal produjo el 75\% de las tesis y, dentro de ella, las disciplinas de Parasitología (64/ 247) y Epidemiología (46/247) fueron las más estudiadas. Los laboratorios de Microbiología y Parasitología (37\%), Patología Aviar (12\%), Medicina Veterinaria Preventiva (8.5\%) y Reproducción y Obstetricia Veterinaria (7.7\%) fueron los que tuvieron un mayor número de tesis. Así mismo, tres docentes tuvieron a su cargo la dirección del 33\% y ocho docentes del $57 \%$ de ellas. Se detectó asimetrías en algunas unidades académicas entre la proporción de docentes que participan como directores y como jurados de tesis recomendándose medidas correctivas.
\end{abstract}

Palabras clave : tesis, bibliometría, investigación

\section{Abstract}

Undergraduate thesis play a major role on research activities at the Veterinary Faculty of Universidad Nacional Mayor de San Marcos (FMV-UNMSM) and therefore, this study was planned to evaluate the research component through a bibliometric analysis of all theses that were defended during the 2001-2006 period. Analyzed data included the academic unit, discipline, animal species, adviser and thesis committee. Data was organized

\footnotetext{
${ }^{1}$ Laboratorio de Bioquímica, Nutrición y Alimentación Animal, Facultad de Medicina Veterinaria, Universidad Nacional Mayor de San Marcos, Lima

${ }^{2}$ Email: fsanmartinh47@gmail.com
} 
and normalized in the institutional database for recording scientific publications (REGULVS) and MS Excel was used to evaluate and build a matrix of coincidences using the macro Microsoft Excel Toolinf $\odot$. A total of 247 theses were registered with an annual average of 41. Canine (15.5\%), bovine (15.5\%), poultry (13.4\%), swine (12.6\%) and alpaca $(11.3 \%)$ were the most studied species. Seventy five percent of the theses were in the area of Animal Health, and among them, Parasitology (64/247) and Epidemiology (46/247) were the most studied disciplines. The laboratories of Microbiology and Parasitology (37\%), Avian Pathology (12\%), Preventive Veterinary Medicine (8.5\%), and Reproduction and Veterinary Obstetrics $(7.7 \%)$ generated most of the theses. Three lecturers were responsible for $33 \%$ of the thesis and eight of them for $57 \%$. Asymmetry was detected among the number of advisers and participants of theses committees, so corrective measures were recommended.

Key words: thesis, bibliometric, research

\section{INTRODUCCIÓN}

Se han realizado pocos estudios bibliométricos en tesis de pregrado en países de habla hispana. Entre ellos se encuentra el trabajo de Quintana (2006) quien evaluó las tesis de la Facultad de Psicología de la Universidad Nacional Mayor de San Marcos (UNMSM) en el Perú; el de Jiménez (2004) quién evaluó las tesis de las facultades de Educación de las universidades Simón Rodríguez y Central de Venezuela; el de Delgado et al. (2006) quienes evaluaron las tesis de la Facultad de Odontología de la Universidad de Córdova; y el de Campos et al. (2005) donde se evaluaron las tesis de pregrado en España durante el periodo 1965 al 2006. En todos estos estudios se valoró principalmente la participación de los estudiantes en la investigación y las tendencias de estos estudios.

La situación de las tesis de pregrado en la Facultad de Medicina Veterinaria (FMV) de la UNMSM fue tratada por San Martín y García (2006), quienes describieron la importancia de la tesis en dicha institución y los esfuerzos para concebirla como un trabajo de investigación universitario en su sentido más amplio; es decir, como un trabajo en equipo entre el maestro (docente) y el discípulo (estudiante), representando, de alguna manera, uno de los más importantes productos de la actividad científica de la Facultad.
La tesis es, entonces para la FMV, el primer paso serio que tienen los jóvenes egresados para incursionar en el campo de la investigación, además de ser requisito para obtener su título de Médico Veterinario. De esta manera, para los intereses de la Facultad, la ejecución de la tesis satisface los objetivos de generar conocimiento a través de la investigación, además de ser una herramienta adicional en la formación académica profesional del estudiante. Por otro lado, la ejecución de las tesis también está enmarcada en procesos administrativos que deben ser monitoreados y evaluados, a fin de hacerla un proceso fluido y eficiente.

Los indicadores bibliométricos permiten evaluar diversos aspectos de la actividad de investigación, distinguiendo áreas y disciplinas del conocimiento, instituciones, países, regiones, etc. Es así que se puede evaluar, por ejemplo, el crecimiento de un campo de la ciencia según el número de trabajos en función al tiempo; o la productividad de los autores por laboratorio o centro de trabajo (Sancho, 1990). Dada la importancia de las tesis en la FMV-UNMSM, era de enorme trascendencia realizar un análisis bibliométrico que permita caracterizarlas y tomar las medidas adecuadas para mejorar sus procesos. En base a esto, se planteó este estudio a fin de evaluar mediante un análisis bibliométrico de las tesis de pregrado que hayan sido sustentadas en un periodo de seis años tomando en 
consideración las variables autor, unidad académica, disciplina, especie animal y jurado de tesis.

\section{Materiales y Métodos}

El presente estudio es un análisis descriptivo, longitudinal y retrospectivo, mediante el uso de herramientas bibliométricas, de las tesis de pregrado ejecutadas, sustentadas e impresas en la FMV-UNMSM durante el periodo 2001 - 2006.

\section{Características Académicas de la FMV- UNMSM}

En el periodo de estudio, la FMV contó en promedio, con cerca de 100 docentes y 500 estudiantes a nivel de pregrado y 100 a nivel de postgrado. En el año 2006, 86 de los 102 docentes fueron a dedicación exclusiva, 11 a tiempo completo y cinco a tiempo parcial; así mismo, 10, 36 y 5 docentes tenían grado de Doctor o Magíster, o título de segunda especialidad, respectivamente. Con respecto a la situación laboral, 78 eran docentes permanentes ( 31 en la categoría Principal, 25 en la categoría de Asociado y 22 en la de Auxiliar) y el resto era contratado por periodos fijos.

La FMV suscribió en 1998, las políticas y estrategias de investigación de la UNMSM (CSI, 1998), declarando a la investigación como una actividad prioritaria. Para ello, cuenta con el apoyo del Instituto Veterinario de Investigaciones Tropicales y de Altura (IVITA) con sus cuatro estaciones experimentales (en Huancayo, Maranganí, Pucallpa e Iquitos), además de laboratorios y clínicas en su sede en Lima. La FMV definió, a inicios de la década pasada, el rol de las tesis como herramienta para la generación de conocimiento, así como para la capacitación de los estudiantes en las actividades propias del ejercicio de la profesión. Para ello, se tomaron medidas académicas y administrativas tendientes a reforzar el proceso de la ejecución de tesis (San Martín y García, 2006).
En los últimos años, una gran cantidad de tesis han sido financiadas por los laboratorios, clínicas o estaciones de la FMV, donde se ejecutan las tesis, y que cuentan con proyectos financiados por la misma universidad, o por entidades nacionales e internacionales; sin embargo, también se desarrollan tesis que tienen financiamiento compartido entre la unidad académica y el tesista.

\section{Clasificación de la Información}

Se revisaron las actas de sustentación de tesis de la Escuela Académica Profesional recabándose la siguiente información: título, nombre del director, año de sustentación y miembros del jurado. En base al director de la tesis se adjudicó la investigación a una unidad académica (laboratorio, clínica o estación experimental) y en base al título se estratificaron por especie animal, por áreas del conocimiento (salud animal, producción animal, ciencias básicas y salud pública), y por disciplina.

\section{Procesamiento de Datos}

Los datos fueron incluidos en Registro Único de Literatura Veterinaria en San Marcos (REGULVS), base de datos que registra las comunicaciones científicas de la FMV-UNMSM desde su creación en 1946. El REGULVS está hecho en base al programa Procite 5.03, utilizado en estudios de tipo bibliométrico, que permite la normalización de los documentos y conteos simples de la información.

Los datos se colocaron primeramente en Microsoft ${ }^{\circledR}$ Office Excel y de allí exportados manualmente a los campos del REGULVS. Los datos, una vez organizados y normalizados, fueron exportados nuevamente a Excel para su evaluación, y finalmente se confeccionaron las matrices de coincidencia (Ejemplo, laboratorio - año) utilizando la macro de Microsoft Excel Toolinfœ. 


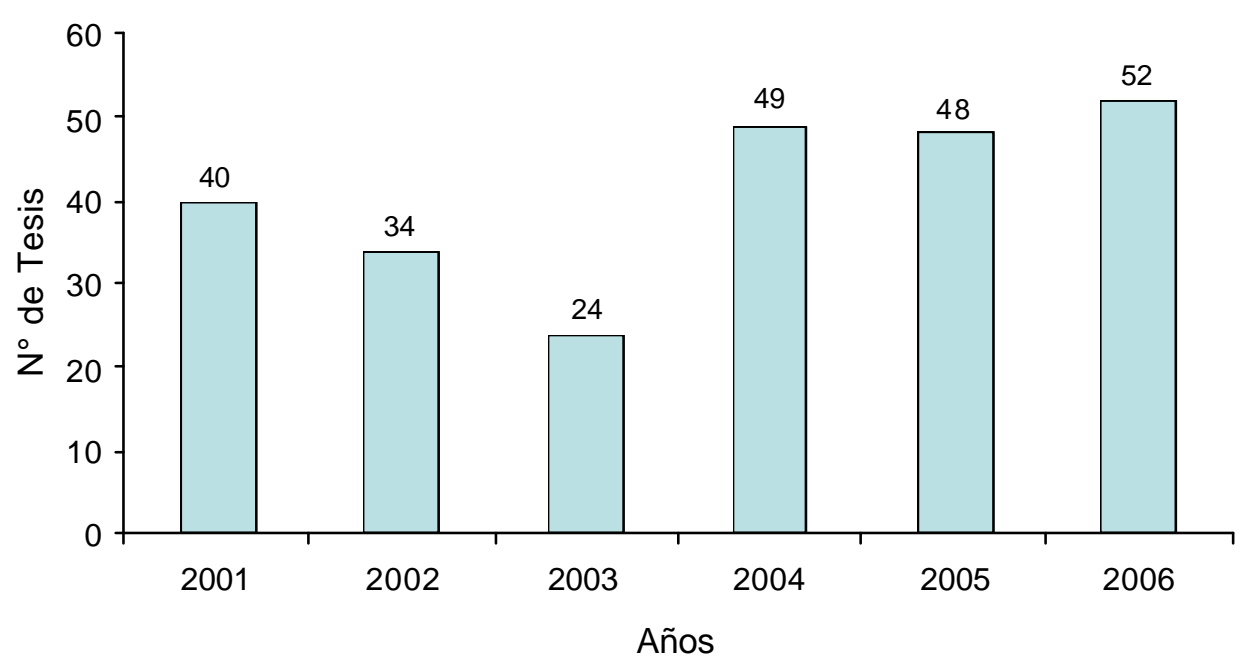

Figura 1. Número de tesis de pregrado sustentadas en la Facultad de Medicina Veterinaria de la UNMSM en el periodo 2001-2006

\section{Resultados y Discusión}

El número de tesis producidas en el periodo 2001 - 2006 se muestra en la Fig. 1, el cual difiere ligeramente de lo reportado por San Martín y García (2006) quienes tomaron la información de los Anuarios Estadísticos de la Universidad. Se registraron 247 tesis con una media anual de 41. Este promedio tendió a incrementarse en los últimos tres años llegando a una media anual de 50. Esta producción de tesis representa el $25 \%$ de las tesis producidas en las 51 escuelas académicas de la UNMSM (UNMSM, 2001, 2002, 2003, 2004 y 2005).

El mayor número de tesis involucró al canino (38) y al bovino (38) como sujeto de estudio, seguidas por las aves (33), porcinos (31), alpacas (28), y otras especies en menor grado (Cuadro 1). Las especies tradicionales de investigación han sido los bovinos y los caninos; sin embargo, las tesis en las cuatro especies de camélidos sudamericanos suman 38. Este alto número de trabajos de tesis de pregrado observado en estas especies coincide por lo reportado por Rojas (1995) para trabajos de investigación que fueron publicados por los profesores de la FMV-UNMSM, y es una clara indicación de la prioridad que viene teniendo este grupo de especies en las actividades de investigación de la Facultad.

El creciente interés en camélidos sudamericanos como entes de investigación se sustenta en la existencia de una estación experimental dedicada a estas especies (Maranganí-La Raya) y en la dedicación de seis de los ocho docentes con grado de doctor a trabajos de investigación con estos animales. Además, la Facultad, desde su creación, ha considerado a los camélidos sudamericanos como especies únicas y estratégicas del país, que se localizan en zonas deprimidas y en manos de comunidades campesinas de escasos recursos tecnológicos y económicos, lo que conlleva a su vez, a la existencia de recursos económicos disponibles para proyectos de desarrollo e investigación.

Dentro de las mascotas, el perro es la especie predominante como sujeto de estudio en tesis de pregrado (38) en comparación con el gato (2), y supera individualmente a todas las especies estudiadas, con excepción del bovino. La preferencia de los estudiantes para dedicarse a la clínica de mascotas en su desempeño profesional, así como una relativa facilidad para realizar tesis con estos 
Cuadro 1. Número de tesis de pregrado sustentadas en la Facultad de Medicina Veterinaria de la UNMSM en el periodo 2001-2006, según la especie animal bajo estudio

\begin{tabular}{|c|c|c|c|c|c|c|c|}
\hline \multirow{2}{*}{ Especie } & \multicolumn{6}{|c|}{ Año } & \multirow{2}{*}{ Total } \\
\hline & 2001 & 2002 & 2003 & 2004 & 2005 & 2006 & \\
\hline Canino & 4 & 7 & 5 & 6 & 8 & 8 & 38 \\
\hline Bovino & 8 & 5 & 4 & 9 & 3 & 9 & 38 \\
\hline Aves & 6 & 4 & 5 & 2 & 9 & 7 & 33 \\
\hline Porcino & 4 & 4 & 1 & 8 & 9 & 5 & 31 \\
\hline Alpaca & 5 & 4 & 3 & 7 & 1 & 8 & 28 \\
\hline Silvestres & 2 & 0 & 0 & 3 & 8 & 2 & 15 \\
\hline Llama & 1 & 1 & 2 & 2 & 2 & 4 & 12 \\
\hline Ovino & 3 & 1 & 0 & 2 & 4 & 0 & 10 \\
\hline Menores $^{1}$ & 1 & 3 & 2 & 2 & 1 & 0 & 9 \\
\hline Equino & 1 & 2 & 0 & 1 & 1 & 1 & 6 \\
\hline Caprino & 1 & 0 & 0 & 3 & 0 & 1 & 5 \\
\hline Humano & 0 & 1 & 0 & 0 & 0 & 2 & 3 \\
\hline Vicuña & 0 & 1 & 0 & 0 & 0 & 1 & 2 \\
\hline Felino & 0 & 0 & 1 & 1 & 0 & 0 & 2 \\
\hline Guanaco & 0 & 0 & 0 & 0 & 0 & 1 & 1 \\
\hline Otros & 2 & 0 & 0 & 0 & 0 & 1 & 13 \\
\hline
\end{tabular}

${ }^{1}$ Cuy, conejo, rata, ratón

animales, explica el elevado número de tesis en esta especie; a diferencia de la frecuencia de trabajos de investigación llevados a cabo por docentes de la Facultad, donde el perro solo representó el $1.9 \%$ de 948 resúmenes de investigación (Rojas, 1995).

$\mathrm{Al}$ analizar las tesis por áreas y disciplinas se obtuvo un total de 323 registros, pese a que el número de tesis fue de 247 , debido a que una tesis puede abarcar más de una disciplina (Cuadro 2). El área que más tesis ha producido fue la de Salud Animal con el 75\%, seguido por Producción animal con 17\%. Esta proporción es diferente a la que presenta Rojas (1995) al evaluar los resúmenes de investigación publicados en los 30 años de creación del Centro de Investigación IVITA, donde el área de Producción Animal representó la tercera parte de los trabajos de investigación realizados. La predominancia actual del área de Salud Animal se explica por el mayor número de docentes investigadores en esta área y, el reforzamiento, en los últimos años, de los aspectos médicos de la profesión a expensas del área de Producción Animal.
Dentro del área de Salud Animal predominó la disciplina de Parasitología con el $26 \%$ (64/242), con una media anual sostenida de 10 tesis, seguida de las disciplinas de Epidemiología con un 19\%, y Bacteriología y Virología con $16 \%$ cada una; en tanto que la disciplina de Reproducción predominó con un $39 \%$ dentro del área de Producción Animal.

En el Cuadro 3 se muestra el número de tesis de pregrado en base a la unidad académica responsable de su ejecución, sean estas laboratorios, clínicas o estaciones experimentales. El Laboratorio de Microbiología y Parasitología Veterinaria tuvo la responsabilidad del mayor número de tesis (37\%), seguido por el de Patología Aviar con $12 \%$, Medicina Preventiva con $8.5 \%$ y Reproducción y Obstetricia Veterinaria con 7.7\%. Asimismo, las clínicas de Animales Mayores y Menores en conjunto produjeron el $8.5 \%$ de las tesis. Por otro lado, las estaciones experimentales de El Mantaro e Iquitos produjeron un número reducido de tesis, en tanto que las de Pucallpa y 
Cuadro 2. Número de tesis de pregrado sustentadas en la Facultad de Medicina Veterinaria de la UNMSM en el periodo 2001-2006, según el área del conocimiento y la disciplina identificada

\begin{tabular}{|c|c|c|c|c|c|c|c|}
\hline \multirow{2}{*}{$\begin{array}{l}\text { Área del conocimiento y } \\
\text { disciplina }\end{array}$} & \multicolumn{6}{|c|}{ Año } & \multirow{2}{*}{ Total } \\
\hline & 2001 & 2002 & 2003 & 2004 & 2005 & 2006 & \\
\hline Salud animal & & & & & & & 242 \\
\hline Parasitología & 8 & 11 & 12 & 13 & 10 & 10 & 64 \\
\hline Epidemiología & 4 & 9 & 8 & 9 & 7 & 9 & 46 \\
\hline Virología & 8 & 4 & 4 & 4 & 9 & 10 & 39 \\
\hline Bacteriología & 4 & 5 & 1 & 12 & 7 & 10 & 39 \\
\hline Inmunología & 0 & 0 & 3 & 5 & 6 & 7 & 21 \\
\hline Histopatología & 2 & 1 & 1 & 2 & 1 & 6 & 13 \\
\hline Patología clínica & 2 & 0 & 1 & 3 & 4 & 2 & 12 \\
\hline Cirug ía & 0 & 2 & 0 & 1 & 1 & 0 & 4 \\
\hline Radiología & 0 & 1 & 0 & 0 & 0 & 2 & 3 \\
\hline Sanidad & 0 & 0 & 0 & 0 & 0 & 1 & 1 \\
\hline Producción Animal & & & & & & & 54 \\
\hline Reproducción & 8 & 2 & 1 & 5 & 3 & 2 & 21 \\
\hline Nutrición & 0 & 7 & 1 & 1 & 3 & 1 & 13 \\
\hline Producción animal & 4 & 0 & 0 & 2 & 3 & 4 & 13 \\
\hline Producción avícola & 0 & 1 & 2 & 0 & 2 & 2 & 7 \\
\hline Ciencias Básicas & & & & & & & 14 \\
\hline Farmacología & 1 & 0 & 1 & 0 & 4 & 0 & 6 \\
\hline Fisiología & 2 & 0 & 0 & 2 & 1 & 1 & 6 \\
\hline Anatomía & 1 & 0 & 0 & 0 & 1 & 0 & 2 \\
\hline Genética & 0 & 0 & 0 & 1 & 0 & 1 & 2 \\
\hline Salud Pública & & & & & & & 11 \\
\hline Salud Pública & 2 & 0 & 2 & 4 & 1 & 1 & 10 \\
\hline Zoonosis & 0 & 0 & 0 & 0 & 1 & 0 & 1 \\
\hline Total & 46 & 43 & 37 & 64 & 64 & 69 & 323 \\
\hline
\end{tabular}

Maranganí-La Raya tuvieron una producción nula. Este bajo número o ausencia de tesis en las estaciones experimentales del IVITA se explica por la lejanía de las estaciones con relación a la sede de la Facultad en Lima, que dificulta que los investigadores del IVITA actúen como directores de tesis; sin embargo, son varias las tesis que se desarrollan en dichas estaciones pero bajo la dirección de algún docente con sede en alguno de los laboratorios de la Facultad.
En el Cuadro 4 se muestra el número de tesis que estuvo bajo la responsabilidad de cada docente. Se observa que la Dra. Chávez (17\%) seguida de la Dra. Icochea (9\%) y la Dra. Rivera (7\%) produjeron la tercera (33\%) parte de la totalidad de tesis durante el periodo bajo estudio. Así mismo, el 57\% de las tesis fueron dirigidas por ocho docentes, donde cada uno llegó a dirigir 10 ó más tesis. Sancho (1990) señala que la productividad de los autores está en función de una serie 
Cuadro 3. Número de tesis de pregrado sustentadas en la Facultad de Medicina Veterinaria de la UNMSM en el periodo 2001-2006, según la unidad académica responsable del estudio

\begin{tabular}{|c|c|c|c|c|c|c|c|}
\hline \multirow{2}{*}{ Unidad Académica } & \multicolumn{6}{|c|}{ Años } & \multirow{2}{*}{ Total } \\
\hline & 2001 & 2002 & 2003 & 2004 & 2005 & 2006 & \\
\hline \multicolumn{8}{|l|}{ Laboratorio } \\
\hline Microbiol. y Parasitol. Vet. & 16 & 16 & 11 & 16 & 17 & 15 & 91 \\
\hline Patología Aviar & 4 & 2 & 5 & 2 & 10 & 7 & 30 \\
\hline Med. Vet. Preventiva & 2 & 2 & - & 5 & 3 & 9 & 21 \\
\hline Reproducción y Obst. Vet. & 5 & 2 & 1 & 5 & 3 & 3 & 19 \\
\hline Salud Pública y Salud Animal & 2 & - & 2 & 4 & 2 & 3 & 13 \\
\hline Histología, Emb. y Pat. Vet. & - & - & 2 & 2 & 2 & 6 & 12 \\
\hline Bioq., Nutr. y Alim. Animal & - & 6 & 1 & 1 & 3 & - & 11 \\
\hline Patología Clínica & 2 & 1 & - & 2 & 3 & 2 & 10 \\
\hline Fisiología Animal & 2 & - & - & 3 & 1 & - & 6 \\
\hline Farmacología y Toxicol. Vet. & 1 & - & 1 & - & 1 & - & 3 \\
\hline Zootecnia y Prod. Agropec. & 2 & - & - & - & - & 1 & 3 \\
\hline Prod. Avícola y Esp. Menores & 1 & 2 & - & - & - & - & 3 \\
\hline Anat. Anim. y Fauna Silvestre & 1 & - & - & - & 1 & - & 2 \\
\hline \multicolumn{8}{|l|}{ Clínica } \\
\hline Animales Menores & 1 & 3 & - & 1 & 1 & 4 & 10 \\
\hline Animales Mayores & 1 & - & 1 & 6 & 1 & 2 & 11 \\
\hline \multicolumn{8}{|l|}{ Estación Experimental } \\
\hline El Mantaro & - & - & - & 1 & - & - & 1 \\
\hline Iquitos & - & - & - & 1 & - & - & 1 \\
\hline Total & 40 & 34 & 24 & 49 & 48 & 52 & 247 \\
\hline
\end{tabular}

de variables que se pueden agrupar en dos categorías: a) características personales, como inteligencia, perseverancia y capacidad, entre otras, y b) medio ambiente como sueldo, financiamiento, facilidades, disciplina en la que investiga y colegas con los que se interactúa, entre otros. A estas dos categorías se podría agregar, para el caso de las tesis, la influencia o empatía del docente sobre los estudiantes para que se inclinen a solicitar su participación como director.

Se tuvo una media anual de 96 docentes, pero únicamente 44 de ellos dirigieron alguna tesis durante el periodo de estudio
(Cuadro 4). Esto implicó que cada director de tesis tuvo bajo su responsabilidad un promedio de 2.3 tesis por año, aunque siempre con una gran variabilidad entre docentes. En el Cuadro 5 se presenta la relación encontrada entre el número de tesis sustentadas y el número de directores de tesis, donde se denota una alta concentración de tesis bajo la responsabilidad de un número reducido de docentes.

En el Cuadro 6 se observa la relación entre el número acumulado de directores y miembros de jurado de tesis por unidad académica. Con el fin de nivelar la relación Di- 
Cuadro 4. Número de tesis de pregrado dirigidas por docentes y número de docentes directores de tesis en la Facultad de Medicina Veterinaria de la UNMSM en el periodo 2001-2006

\begin{tabular}{lccccccc}
\hline Docente & 2001 & 2002 & 2003 & 2004 & 2005 & 2006 & Total \\
\hline Amanda Chávez Velásquez & 4 & 8 & 10 & 6 & 9 & 4 & 41 \\
Eliana Icochea D`Arrigo & 4 & 2 & 3 & 1 & 6 & 6 & 22 \\
Hermelinda Rivera Gerónimo & 6 & 6 & - & 1 & 1 & 4 & 18 \\
Armando González Zariquey & 2 & 2 & - & 5 & 2 & 4 & 15 \\
Miguel Vilca López & 2 & - & 2 & 4 & 2 & 3 & 13 \\
Alfredo Delgado Castro & 1 & - & 1 & 6 & 1 & 2 & 11 \\
Raúl Rosadio Alcántara & 3 & 1 & 1 & 3 & - & 2 & 10 \\
Sonia Calle Espinoza & 1 & 1 & - & 2 & 5 & 1 & 10 \\
Olga Lí Elías & 1 & - & - & 2 & 4 & 2 & 9 \\
Mónica Alba Chincha & - & - & 2 & 1 & 3 & 1 & 7 \\
Víctor Leyva Vallejos & 3 & 2 & - & - & - & 2 & 7 \\
Wilfredo Huanca López & - & - & - & 5 & 1 & 1 & 7 \\
Alfonso Chavera Castillo & - & - & 2 & 1 & 1 & 2 & 6 \\
Alberto Manchego Sayán & - & - & - & 1 & 2 & 2 & 5 \\
Diego Díaz Coahila & - & - & - & - & 1 & 4 & 5 \\
Felipe San Martín Howard & - & 3 & 1 & - & 1 & - & 5 \\
Nieves Sandoval Chaupe & - & - & - & - & 1 & 4 & 5 \\
Víctor Fernández Anhuamán & 1 & 3 & - & 1 & - & - & 5 \\
Sergio Cueva Moreno & 1 & - & - & 2 & 1 & - & 4 \\
Teresa Arbaiza Fernández & - & 3 & - & - & 1 & - & 4 \\
Teresa López Urbina & 1 & - & - & 3 & - & - & 4 \\
Edgardo Figueroa Terry & 1 & 2 & - & - & - & - & 3 \\
Luisa Echevarría Cureé & 2 & - & 1 & - & - & - & 3 \\
Otros docentes & 7 & 1 & 1 & 5 & 6 & 8 & 28 \\
\hline Total de tesis & 40 & 34 & 24 & 49 & 48 & 52 & 247 \\
\hline Número de docentes directores & 22 & 12 & 10 & 21 & 22 & 22 & 44 \\
\hline Número de docentes por año & 96 & 89 & 91 & 94 & 105 & 102 & 96 \\
\hline
\end{tabular}

rector/Miembro de Jurado de Tesis, el número de directores se multiplicó por el factor 3 . Esta relación, indica que aquella unidad académica con cociente cercano a uno es una unidad que tiene un equilibrio entre la participación de docentes directores y docentes jurados en el proceso de la ejecución de la tesis, como es el caso del Laboratorio de Patología Clínica y la Clínica de Animales Mayores; cocientes superiores a uno $(>1)$ indican un predominio de docentes directores sobre docentes jurados, como es el caso de los laboratorios de Patología Aviar, Microbiología y Parasitología, y Reproducción y Obstetricia Veterinaria; en tanto que cocientes menores a uno $(<1)$ indican un desequilibrio indeseable, como es el caso de los laboratorios de Zootecnia y Producción Agropecuaria, Fisiología Animal, e Histología, Embriología y Patología Veterinaria. Este desequilibrio debe ser corregido a fin de evitar que docentes que no pasen con frecuencia por la experiencia de dirigir una tesis sean evaluadores o 
Cuadro 5. Relación entre el número de tesis de pregrado y el número de directores de tesis en la Facultad de Medicina Veterinaria de la UNMSM en el periodo 2001-2006

\begin{tabular}{cccc}
\hline \multirow{2}{*}{$\begin{array}{c}\mathrm{N}^{\circ} \text { de tesis por } \\
\text { director de tesis }\end{array}$} & $\begin{array}{c}\text { Directores } \\
(\mathrm{n})\end{array}$ & $\mathrm{n}$ & Tesis \\
\cline { 3 - 4 } & 14 & 14 & $\%$ \\
\hline 1 & 17 & 57 & 23.7 \\
$2-5$ & 10 & 95 & 38.5 \\
$6-15$ & 3 & 81 & 32.8 \\
\hline 15 & 44 & 247 & 100.0 \\
\hline
\end{tabular}

Cuadro 6. Relación entre el número de directores de tesis y el número de miembros del Jurado de Tesis en la Facultad de Medicina Veterinaria de la UNMSM $^{1}$ en el periodo 2001-2006, según la unidad académica

\begin{tabular}{|c|c|c|c|}
\hline \multirow[b]{2}{*}{ Unidad Académica } & \multicolumn{2}{|c|}{$\mathrm{N}^{\circ}$ acumulado de docentes } & \multirow{2}{*}{$\begin{array}{c}\text { Relación } \\
\text { Jurado/Director }\end{array}$} \\
\hline & Directores & $\begin{array}{l}\text { Miembros } \\
\text { de Jurado }\end{array}$ & \\
\hline
\end{tabular}

Laboratorio

Patología Aviar

Microbiol. y Parasitol. Veterinaria

Reproducción y Obstetricia Vet.

Bioquímica, Nutr. y Alim. Animal

Salud Pública y Salud Animal

Farmacología y Toxicología Vet.

Patología Clínica

Medicina Veterinaria Preventiva

Anatomía Animal y Fauna Silvestre

Prod. Avícola y Especies Menores

Histología, Embriología y Patol. Vet.

Fisiología Animal

Zootecnia y Producción Agropecuaria

$\begin{array}{ccc}30 & 18 & 5.0 \\ 91 & 131 & 2.1 \\ 19 & 31 & 1.8 \\ 11 & 21 & 1.6 \\ 13 & 26 & 1.5 \\ 3 & 6 & 1.5 \\ 10 & 30 & 1.0 \\ 21 & 113 & 0.6 \\ 2 & 10 & 0.6 \\ 3 & 19 & 0.5 \\ 12 & 91 & 0.4 \\ 6 & 41 & 0.4 \\ 3 & 123 & 0.1\end{array}$

Clínica

$\begin{array}{llll}\text { Animales Mayores } & 11 & 31 & 1.1 \\ \text { Animales Menores } & 10 & 48 & 0.6\end{array}$

\begin{tabular}{lll}
\hline Total & 245 & 739 \\
\hline 1 & No se incluyen las estaciones experimentales del IVITA, debido a su escasa participación como \\
directores de tesis o miembros de jurado de tesis. \\
2 \\
El número de directores se multiplica por 3 para nivelar la relación de 3 miembros de jurado por un \\
director en la conformación del Jurado de Tesis
\end{tabular}


jurados de las mismas.

El cociente bajo del Laboratorio de Medicina Veterinaria Preventiva se explica por la activa participación de sus docentes como miembros de jurados de tesis, dado que sus docentes son especialistas en el área de estadística, y como tales, son constantemente requeridos para verificar la calidad de los diseños experimentales y de los análisis estadísticos realizados (San Martín, 2006).

\section{Conclusiones}

? Las principales especies que fueron sujeto de estudio en tesis de pregrado en la Facultad de Medicina Veterinaria de la UNMSM fueron los caninos (15.5\%) y los bovinos (15.5\%), seguidos por las aves $(13.4 \%)$, porcinos $(12.6 \%) \mathrm{y}$ alpacas $(11.3 \%)$.

? El área de Salud Animal produjo el 75\% de las tesis de pregrado, y dentro de esta área, las disciplinas de Parasitología (64/ 247) y Epidemiología (46/247) fueron las más estudiadas.

? Los laboratorios de Microbiología y Parasitología (37\%), Patología Aviar (12\%), Medicina Veterinaria Preventiva (8.5\%) y Reproducción y Obstetricia Veterinaria $(7.7 \%)$ fueron los laboratorios que tuvieron la responsabilidad del mayor número de tesis.

? Tres docentes tuvieron a su cargo la dirección del 33\% de las tesis de pregrado sustentadas (ocho docentes del 57\%) dentro de un promedio anual de 98 docentes.

? $\quad$ Los docentes de algunas unidades académicas no participaron como directores de tesis pero, en cambio, fueron considerados como miembros de jurado de tesis.

\section{Literatura Citada}

1. Camps D, Recuero Y, María Samar E, Ávila R. 2005. Análisis bibliométrico de tesis de doctorado del área de las Ciencias de la Salud: primera parte, Odontología. Rev Fac Cienc Médicas Univ Nac Córdoba 62(3):53-56.

2. [CSI] Consejo Superior de Investigaciones. 1998. Política y estrategia de la investigación en la Universidad Nacional Mayor de San Marcos. (Perú). Lima: UNMSM. $47 \mathrm{p}$.

3. Delgado López-Cózar E, Torres-Salinas D, Jiménez-Contreras E, RuízPérez R. 2006. Análisis bibliométrico y de redes sociales aplicado a las tesis bibliométricas defendidas en España (1976-2002): temas, escuelas científicas y redes académicas. Rev Esp Doc Cient 29(4): 493-524.

4. Jiménez E. 2004. Análisis bibliométrico de tesis de pregrado de estudiantes venezolanos en el área educación: 1990 1999. Rev Iberoamericana Educ [Internet], Disponible en: http:// www.rieoei.org/deloslectores/623 Jimenez. PDF

5. Rojas M. 1995. IVITA: 30 Años de ciencia y tecnología pecuaria peruana. Lima: UNMSM. 267 p.

6. Sancho, $R$. 1990. Indicadores bibliométricos utilizados en la evaluación de la ciencia y la tecnología. Revisión bibliográfica. Rev Esp Doc Cient 13(34): $842-865$.

7. San Martín F y García M. 2006. La tesis y su problemática en la Facultad de Medicina Veterinaria de la UNMSM. Rev Inv Vet, Perú 17(1): 81-88.

8. San Martín F. 2006. Medicina Veterinaria 2006. Lima: UNMSM. 319 p.

9. [UNMSM] Universidad Nacional Mayor de San Marcos. Compendio estadístico. 2001. Lima: Unidad de Estadística e Informática de la Oficina General de Planificación, UNMSM. 283 p. 
10. [UNMSM] Universidad Nacional Mayor de San Marcos. Compendio estadístico.. 2002. Lima: Unidad Estadística e Informática de la Oficina General de Planificación, UNMSM. 274 p.

11. [UNMSM] Universidad Nacional Mayor de San Marcos. Compendio estadístico. 2003. Lima: Unidad Estadística e Informática de la Oficina General de Planificación, UNMSM. 381 p.
12. [UNMSM] Universidad Nacional Mayor de San Marcos. Compendio estadístico. 2004. Lima: Unidad Estadística e Informática de la Oficina General de Planificación, UNMSM. 485 p.

13. [UNMSM] Universidad Nacional Mayor de San Marcos. Compendio estadístico. 2005. Lima: Unidad Estadística e Informática de la Oficina General de Planificación, UNMSM. 485 p. 